Case Report

\title{
Chronic pseudoaneurysm of the left ventricle
}

\author{
Timo Baks ${ }^{1,2}$, Filippo Cademartiri ${ }^{1,2}$, Han A. M. Spierenburg ${ }^{3}$ \& Pim J. de Feyter ${ }^{1,2}$ \\ ${ }^{1}$ Department of Cardiology, Thoraxcenter, Erasmus MC, University Medical Center, Rotterdam, \\ The Netherlands; ${ }^{2}$ Department of Radiology, Erasmus MC, University Medical Center, Rotterdam, \\ The Netherlands; ${ }^{3}$ Department of Cardiology, Vlietland Hospital Schiedam, Rotterdam, The Netherlands
}

Received 24 June 2005; accepted in revised form 16 July 2005

Key words: cardiovascular magnetic resonance imaging, chronic myocardial infarction, left ventricular pseudoaneurysm, multislice computed tomography

\begin{abstract}
We present a case of a 55-year-old men who suffered a silent myocardial infarction four years earlier and presented with exertional dyspnoea. Cardiac magnetic resonance imaging (CMR) and Multislice computed tomography (MSCT) was performed and revealed a giant pseudoaneursym of the lateral wall of the left ventricle with the presence of a thrombus in the lateral wall of the pseudoaneursym. We present this case since excellent non-invasive evaluation of the pseudoaneursym was feasible using state-of-the-art imaging modalities. Information on left ventricular geometry and function as well as myocardial viability and coronary anatomy is available when both MSCT and CMR are performed. This combined approach of these two imaging modalities provide clinically relevant information and may guide therapeutic decision making.
\end{abstract}

\section{Introduction}

Formation of a pseudoaneurysm of the left ventricle is a rare complication of acute myocardial infarction and occurs most frequently in the inferior wall. The wall of the pseudoaneurysm contains pericardium and/or scar tissue and not myocardium as is observed in a true aneurysm of the left ventricle [1]. The diagnosis might be difficult to make since a pseudoaneurysm is easily confused with a true aneurysm of the left ventricle [2]. A pseudoaneurysm often needs urgent surgical treatment while a true aneurysm might be treated medically [3]. Advanced non-invasive imaging modalities like cardiac Magnetic Resonance Imaging (CMR) and Multislice Computed
Tomography (MSCT) provide clinically relevant information on coronary anatomy, left ventricular function and myocardial viability [4-6]. We present a patient with a pseudoaneurysm of the left ventricle 4 years after acute myocardial infarction and performed CMR and MSCT to evaluate left ventricular geometry and function as well as myocardial viability and coronary anatomy.

\section{CT protocol}

The examination was performed using a 16-row MSCT scanner (Sensation 16, Siemens, Germany; collimation $16 \times 0.75 \mathrm{~mm}$, rotation time $420 \mathrm{~ms}$, table feed $3.0 \mathrm{~mm} /$ rotation, tube voltage $120 \mathrm{kV}$, 
tube current 400-450 mAs). After intravenous administration of $120 \mathrm{ml}$ of non-ionic contrast material (Visipaque ${ }^{\circledR}$ 320, Amersham Health, UK), an automatic bolus tracking technique triggered the start of the MSCT-scan. Images were reconstructed using retrospective ECG-gating during the mid- to end-diastolic phase to provide nearly motion-free image quality.

\section{CMR protocol}

A clinical 1.5-T CMR scanner with a dedicated cardiac four element phased-array receiver coil was used for imaging (Signa CV/i, GE Medical systems, Milwaukee, Wisconsin USA). Repeated breath-holds and gating to the electrocardiogram were applied to minimize the influence of cardiac and respiratory motion on data collection.

Cine-CMR was performed with a steady-state free-precession technique (FIESTA, GE) using the following imaging parameters: 24 temporal phases per slice; field of view 32 to $36 \times 32$ to $36 \mathrm{~cm}$; rectangular field of view $75-100 \%$; repetition time 3.4 ; time to echo 1.4; flip angle $45^{\circ}$; matrix $192 \times 160$; bandwidth $83 \mathrm{kHz}$; number of averages 0.75 . To cover the entire left ventricle 9 to 12 consecutive slices of $8 \mathrm{~mm}$ were planned in shortaxis view (gap of $2 \mathrm{~mm}$ ) on the four-chamber view of the left ventricle.

Myocardial distribution of delayed enhancement was studied 10-20 min following administration of
Gadolinium-DTPA $\quad(0.2 \mathrm{mmol} / \mathrm{kg}, \quad$ Magnevist ${ }^{\circledR}$, Schering, Germany). A two-dimensional T1weighted inversion recovery gradient-echo sequence with the following imaging parameters was used: field of view 32 to $36 \times 32$ to $36 \mathrm{~cm}$; rectangular field of view 75-100\%; slice thickness $8 \mathrm{~mm}$; gap $2 \mathrm{~mm}$; repetition time 7.3; time to echo 1.6; flip angle $20^{\circ}$; inversion pulse $180^{\circ}$; matrix $256 \times 192$; 1 number of averages; bandwidth $17.9 \mathrm{kHz}$; inversion time $180-300 \mathrm{~ms}$. The inversion time was adjusted to null the signal of remote myocardium. Slice locations were copied from the locations of the cine-images (Figure 1).

\section{Case report}

A 55-year-old men suffered a silent myocardial infarction four years earlier diagnosed by the occurrence of new Q-waves on an electrocardiogram. Recently, he presented with complaints of exertional dyspnoea. A transthoracic echocardiogram was suspicious for a pseudoaneurysm. More detailed anatomy was obtained with cardiovascular magnetic resonance (CMR) imaging and multislice computed tomography (MSCT). CMR revealed a pseudoaneurysm in the lateral wall of the left ventricle with a volume of $351 \mathrm{ml}$ and a diameter of the neck of $1.4 \mathrm{~cm}$. Left ventricular function was depressed (ejection fraction 42\%). A large non contrast-enhanced filling defect was seen at the lateral wall of the pseudoaneurysm indi-
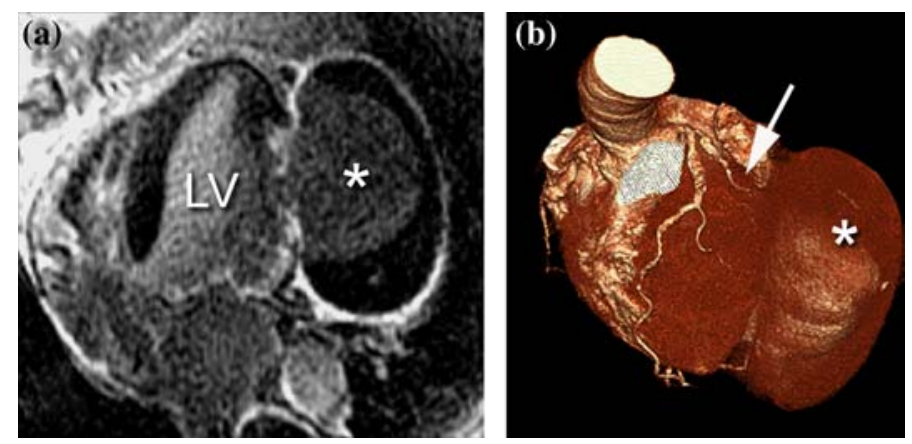

Figure 1. (a) Cardiovascular Magnetic Resonance (1.5 T) performed $15 \mathrm{~min}$ after administration of $0.2 \mathrm{mmol} / \mathrm{kg}$ Gadolinium-DTPA demonstrates a pseudoaneurysm in the lateral wall of the left ventricle; $*=$ pseudoaneurysm; LV $=$ left ventricle. (b) Multislice Computed Tomography (16-slice) demonstrated the three-dimensional reconstruction of the pseudoaneurysm. The left circumflex coronary artery is totally occluded (arrow). 
cating a mural thrombus. The thrombus was surrounded by a bright enhanced rim of pericardium, indicating scar/fibrous tissue. Three-dimensional reconstruction of the MSCT images provided the location and the size of the pseudoaneurysm and an occluded circumflex coronary artery. Surgical intervention was based on the combined information of both imaging modalities and consisted of resection of the pseudoaneurysm with inserting of a pericardial patch and coronary artery bypass grafting. The postoperative course was uneventful and six months after surgery the patient could tolerate exercise well. Left ventricular ejection fraction was assessed with CMR after resection of the pseudoaneurysm and was increased from $42 \%$ to $46 \%$.

\section{Discussion}

CMR and MSCT provide clinically relevant information in patients with a postinfarction pseudoaneurysm of the left ventricle. CMR provides information on left ventricular function and the location and extent of the pseudoaneurysm. Moreover, 10-30 min after administration of gadolinium-derivates, the extent of myocardial viability can be assessed [7]. MSCT provides a three-dimensional data set that can be used for reconstruction to give an overview of the often complicated geometry of the left ventricle and pseudoaneurysm [8]. MSCT also provides information on coronary artery stenosis, although an increased heart rate ( $>70$ beats per min) might hinder evaluation. The combined information on left ventricular geometry and function, myocardial viability and coronary anatomy may guide therapeutic decision making. In the currently presented case, the surgeon was able to resect the pseudoaneurysm with good result.

Other non-invasive imaging modalities have been proposed for the diagnosis of a pseudoaneurysm of the left ventricle. Left ventricular angiography can be helpful, although it is limited by a two-dimensional view of the pseudoaneurysm making assumptions on three-dimensional geom- etry necessary. Radionuclide ventriculography is also used to assess left ventricular geometry, but diagnostic accuracy is limited due to the limited spatial resolution. Echocardiography is commonly used in clinical practice and provides relevant information. However, echocardiography requires a sufficient acoustic window and it might be difficult to differentiate thrombus from myocardium and pericardium. The presence of a thrombus might subsequently lead to an underestimation of the dimensions of the pseudoaneurysm. Contrast echocardiography is a promising technique that may improve image quality. In conclusion, CMR and MSCT are the preferred non-invasive imaging modalities to evaluate patients with a pseudoaneurysm of the left ventricle.

\section{References}

1. Vlodaver Z, Coe JI, Edwards JE. True and false left ventricular aneurysms. Propensity for the altter to rupture. Circulation 1975; 51(3): 567.

2. Frances C, Romero A, Grady D. Left ventricular pseudoaneurysm. J Am Coll Cardiol 1998; 32(3): 557-61.

3. Brown SL, Gropler RJ, Harris KM. Distinguishing left ventricular aneurysm from pseudoaneurysm. A review of the literature.. Chest 1997; 111(5): 1403-9.

4. Mollet NR, Cademartiri F, Krestin GP, et al. Improved diagnostic accuracy with 16-row multi-slice computed tomography coronary angiography. J Am Coll Cardiol 2005; 45(1): $128-32$.

5. Baks T, van Geuns RJ, Biagini E, et al. Recovery of left ventricular function after primary angioplasty for acute myocardial infarction. Eur Heart J 2005; 26(11): 1070-7.

6. Ohnesorge BM, Hofmann LK, Flohr TG, Schoepf UJ. CT for imaging coronary artery disease: defining the paradigm for its application. Int J Cardiovasc Imaging 2005; 21(1): 85104.

7. Kim RJ, Fieno DS, Parrish TB, et al. Relationship of MRI delayed contrast enhancement to irreversible injury, infarct age, and contractile function. Circulation 1999; 100(19) : 1992-2002.

8. Boxt LM. CT anatomy of the heart. Int $\mathbf{J}$ Cardiovasc Imaging 2005; 21(1): 13-27.

Address for correspondence: T. Baks MD, Erasmus MC, Department of Cardiology and Radiology Thoraxcenter, Ca 227a, P.O. Box 2040, 3000 CA Rotterdam, The Netherlands Tel.: +31-10-463-4127; Fax: +31-10-463-4320

E-mail: t.baks@erasmusmc.nl 